\title{
Antibacterial electrospun zein nanofibrous web encapsulating thymol/cyclodextrin-inclusion complex for food packaging
}

\author{
Zeynep Aytac ${ }^{\mathrm{a}}$, Semran Ipek ${ }^{\mathrm{b}}$, Engin Durgun ${ }^{\mathrm{a}}$, Turgay Tekinay ${ }^{\mathrm{c}, \mathrm{d}}$, Tamer Uyar ${ }^{\mathrm{a}, *}$ \\ a Institute of Materials Science E Nanotechnology, UNAM-National Nanotechnology Research Center, Bilkent University, Ankara 06800, Turkey \\ ${ }^{\mathrm{b}}$ Department of Engineering Physics, Istanbul Medeniyet University, Istanbul 34700, Turkey \\ ${ }^{\mathrm{c}}$ Life Sciences Application and Research Center, Gazi University, Ankara 06830, Turkey \\ d Faculty of Medicine, Department of Medical Biology and Genetics, Gazi University, Ankara 06560, Turkey
}

\section{A R T I C L E I N F O}

\section{Article history:}

Received 12 January 2017

Received in revised form 11 April 2017

Accepted 12 April 2017

Available online 18 April 2017

\section{Keywords:}

Electrospinning

Nanofibers

Thymol

Cyclodextrin

Antibacterial activity

Food packaging

Computational modelling

\begin{abstract}
A B S T R A C T
Thymol (THY)/ $\gamma$-Cyclodextrin $(\gamma-\mathrm{CD}$ ) inclusion complex (IC) encapsulated electrospun zein nanofibrous webs (zein-THY $/ \gamma$-CD-IC-NF) were fabricated as a food packaging material. The formation of THY $/ \gamma-$ CD-IC (1:1 and 2:1) was proved by experimental (X-ray diffraction (XRD), thermal gravimetric analysis (TGA), ${ }^{1} \mathrm{H}$ NMR) and computational techniques. THY $/ \gamma$-CD-IC $(2: 1)$ exhibited higher preservation rate and stability than THY $/ \gamma$-CD-IC (1:1). It is worth mentioning that zein-THY/ $\gamma$-CD-IC-NF $(2: 1)$ preserved much more THY as observed in TGA and stability of THY $/ \gamma$-CD-IC (2:1) was higher, as shown by a modelling study. Therefore, much more THY was released from zein-THY/ $\gamma$-CD-IC-NF (2:1) than zein-THY-NF and zein-THY/ $\gamma$-CD-IC-NF (1:1). Similarly, antibacterial activity of zein-THY/ $\gamma$-CD-IC-NF (2:1) was higher than zein-THY-NF and zein-THY/ $\gamma$-CD-IC-NF (1:1). It was demonstrated that zein-THY/ $\gamma$-CD-IC-NF $(2: 1)$ was most effective in inhibiting the growth of bacteria on meat samples. These webs show potential application as an antibacterial food packaging material.
\end{abstract}

(ㄷ) 2017 Elsevier Ltd. All rights reserved.

\section{Introduction}

Cyclodextrins (CDs) are non-toxic and biodegradable cyclic oligosaccharides that are produced by enzymatic degradation of starch. They are composed of $\alpha$-1,4-linked glucopyranose units forming ultimately a truncated cone-like structure. The most intriguing characteristic of CDs is their capability of making noncovalent host-guest inclusion complexes (ICs) with several type of molecules including food additives and essential oils, owing to their relatively hydrophobic cavities. Here, the driving force of the complexation is the substitution of the high-enthalpy water molecules, which occupy the cavity, with a guest molecule of appropriate polarity and dimensions (Del Valle, 2004; Szejtli, 1998). Many studies have been reported in the literature on the use of CDs for food-related applications (Chen \& Liu, 2016; López-de-Dicastillo, Jordá, Catalá, Gavara, \& Hernández-Munoz, 2011; Mallardo et al., 2016; Samperio et al., 2010). Chen and Liu (2016) showed prolonged antimicrobial activity in the presence of CD-IC of mustard essential oil, due to a reduction in the loss of essential oil during preparation and preservation of films (Chen \&

\footnotetext{
* Corresponding author.

E-mail address: tamer@unam.bilkent.edu.tr (T. Uyar).
}

Liu, 2016). In a study of Mallardo et al. (2016), the thermal stability of limonene was improved and slow release of limonene was achieved (Mallardo et al., 2016). Samperio et al. (2010) deduced that CD-ICs increased the solubility of essential oils dramatically up to ten-fold (Samperio et al., 2010). CDs have been employed in scavenging of undesirable food components as well. López-deDicastillo et al. (2011) conducted a study concerning the retention of cholesterol by using polyvinyl alcohol-cyclodextrin composite films (López-de-Dicastillo et al., 2011).

Thymol (THY) (Fig. 1a) is essentially known as a flavour; however it is also an antibacterial and antioxidant compound. It is a monoterpene found in oregano and thyme, but its delivery remains a challenge because of its hydrophobic and volatile nature. Cyclodextrin inclusion complexes of thymol (THY/CD-IC) has been studied to demonstrate their applicability in pork meat systems (Tao, Hill, Peng, \& Gomes, 2014), to prevent oxidation (Mourtzinos, Kalogeropoulos, Papadakis, Konstantinou, \& Karathanos, 2008), and improve meat stability at up to $75 \%$ relative humidity during long storage times (Cevallos, Buera, \& Elizalde, 2010).

Electrospinning has attracted considerable attention in recent years as a versatile method to produce nanofibers having high surface-to-volume ratio and highly porous structure (Greiner \& 


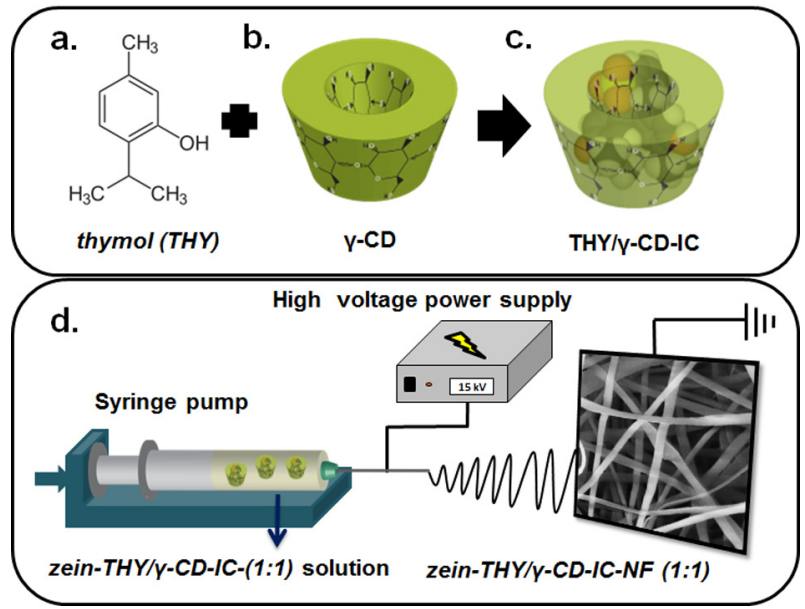

Fig. 1. The chemical structure of (a) THY; (b) schematic representation of $\gamma-C D$, (c) $\mathrm{THY} / \gamma$-CD-IC formation, and (d) electrospinning of nanofibers from zein-THY $/ \gamma$-CDIC $(1: 1)$ solution.

Wendorff, 2007; Ramakrishna, Fujihara, Teo, Lim, \& Ma, 2005). Incorporation of antibacterial agents into packaging material to prevent the proliferation of bacteria is one type of active food packaging. Electrospun nanofibers offer several advantages over films. Firstly, electrospinning can produce nanofibers at room temperature (RT) while polymeric films are usually processed at high temperatures. Therefore, encapsulation of essential oils and flavour/ fragrances which are mostly volatile into electrospun nanofibers is preferable. Secondly, nanofibers are responsive to changes, such as relative humidity and temperature, which makes possible tunable release of the active agents. Hence, electrospun nanofibers have the potential to be used in food packaging applications (Vega-Lugo \& Lim, 2009; Wen, Zhu, Feng et al., 2016; Wen, Zhu, Wu et al., 2016). Moreover, CD-ICs of various compounds including food additives, essential oils, antioxidant and antibacterial agents might be incorporated into electrospun nanofibers for enhancing solubility and thermal stability, controlling release and prolonging shelf life (Aytac \& Uyar, 2016; Aytac, Dogan, Tekinay, \& Uyar, 2014; Aytac, Kusku, Durgun, \& Uyar, 2016a, 2016b; Aytac, Sen, Durgun, \& Uyar, 2015; Aytac et al., 2016; Kayaci \& Uyar, 2012; Uyar, Hacaloglu, \& Besenbacher, 2009; Uyar, Hacaloglu, \& Besenbacher, 2011; Uyar, Nur, Hacaloglu, \& Besenbacher, 2009).

In this study, antibacterial electrospun zein nanofibrous web incorporating CD-IC of thymol was developed as a food packaging material. CD-IC of THY (THY: $\gamma$-CD) was prepared at $1: 1$ and $2: 1$ molar ratio (THY/ $\gamma$-CD-IC (1:1) and THY/ $\gamma$-CD-IC (2:1)) (Fig. 1a-c) and then encapsulated into zein nanofibers by electrospinning (Fig. 1d). $\gamma$-CD was used because it has no side-effects on the absorption of nutrients in food products and nutraceutical applications (Li et al., 2007). The characterization of CD-ICs was carried out using XRD, TGA, and ${ }^{1} \mathrm{H}$ NMR. The computational modelling study was performed in both vacuum and solvent for THY $/ \gamma-\mathrm{CD}$ IC $(1: 1)$ and THY $/ \gamma$-CD-IC $(2: 1)$. Complex-free THY, THY $/ \gamma$-CD-IC $(1: 1)$ and THY $/ \gamma$-CD-IC $(2: 1)$ incorporated zein nanofibers (zeinTHY-NF, zein-THY $/ \gamma$-CD-IC-NF (1:1), zein-THY/ $\gamma$-CD-IC-NF $(2: 1)$ were characterized by scanning electron microscopy (SEM), thermal gravimetric analysis (TGA) and X-ray diffraction (XRD). The release of THY from nanofibers was investigated at different temperatures $\left(37^{\circ} \mathrm{C}, 50^{\circ} \mathrm{C}\right.$, and $75^{\circ} \mathrm{C}$ ) by headspace gas chromatography-mass spectrometry (HS GC-MS). Colony counting method was used to evaluate the antibacterial activity of nanofibers. Lastly, antibacterial activity of nanofibrous webs which were used to pack meat samples was determined throughout 5 days of storage at $4{ }^{\circ} \mathrm{C}$.

\section{Experimental}

\subsection{Materials}

Zein from maize (Sigma-Aldrich), thymol (THY, 98\%, Alfa Aesar), dimethylformamide (DMF, >99\%, Sigma Aldrich), and deuterated dimethylsulfoxide (DMSO- $d_{6}$, deuteration degree min $99.8 \%$ for NMR spectroscopy, Merck), were purchased and used as received without any further purification. The nutrient agar medium (Sigma-Aldrich) that was used for antimicrobial tests contained $0.5 \%$ peptone, $0.1 \%$ meat extract, $0.5 \%$ sodium chloride, $0.2 \%$ yeast extract and $1.5 \%$ agar. Gamma-cyclodextrin $(\gamma-C D)$ was kindly donated by Wacker Chemie AG (Munich, Germany). The water used in the experiments was distilled-deionized from a Millipore Milli-Q ultrapure water system.

\subsection{Preparation of the inclusion complex (IC) $(T H Y / \gamma-C D-I C$ (1:1) and $T H Y / \gamma-C D-I C(2: 1))$}

The formation of solid THY $/ \gamma$-CD-IC (1:1) and THY $/ \gamma-C D-I C$ (2:1) was prepared according to the co-precipitation method. Initially, $\gamma$-CD was dissolved in aqueous solution; then THY was added to $\gamma$-CD solution at $1: 1$ and $2: 1$ ratio (THY: $\gamma-C D$ ). After stirring the solutions overnight at $300 \mathrm{rpm}$, they were kept in a refrigerator $\left(4^{\circ} \mathrm{C}\right)$. After $12 \mathrm{~h}$ the precipitate of THY $/ \gamma$-CD-IC $(1: 1)$ and $\mathrm{THY} / \gamma$-CD-IC (2:1) was collected by filtration.

\subsection{Preparation of electrospinning solutions}

THY without $\gamma$-CD, THY $/ \gamma$-CD-IC $(1: 1)$ and THY $/ \gamma$-CD-IC $(2: 1)$ incorporated zein nanofibers (zein-THY-NF, zein-THY $/ \gamma$-CD-IC-NF $(1: 1)$, and zein-THY $/ \gamma$-CD-IC-NF $(2: 1))$ were produced via the electrospinning technique. For producing zein-THY-NF, THY $(4 \%, \mathrm{w} / \mathrm{w}$, with respect to polymer) was dissolved in DMF at room temperature (RT). Then, $50 \%$ zein $(\mathrm{w} / \mathrm{v})$ was added and zein-THY solution was stirred at $500 \mathrm{rpm}$ for $60 \mathrm{~min}$ before electrospinning. In order to produce zein-THY $/ \gamma$-CD-IC-NF (1:1) and zein-THY $/ \gamma$-CD-IC-NF (2:1), a certain amount of THY $/ \gamma$-CD-IC $(1: 1)$ or $\mathrm{THY} / \gamma-\mathrm{CD}-\mathrm{IC}$ (2:1) corresponding to $4 \% \mathrm{THY}$ ( $\mathrm{w} / \mathrm{w}$, with respect to polymer) was dispersed in DMF at RT. Afterwards, $50 \%$ zein (w/v) was added; the resulting zein-THY/ $\gamma$-CD-IC $(1: 1)$ and zein-THY/ $\gamma$-CD-IC $(2: 1)$ solutions were stirred for $60 \mathrm{~min}$ prior to electrospinning. As a reference sample, $50 \%(\mathrm{w} / \mathrm{v})$ zein solution prepared in DMF was also electrospun (zein-NF). Table S1 summarizes the composition of the zein, zein-THY, zein-THY $/ \gamma$-CD-IC $(1: 1)$, and zein-THY $/ \gamma-C D-$ IC $(2: 1)$ solutions and the morphological findings of electrospun nanofibers; zein-NF, zein-THY-NF, zein-THY/ $\gamma$-CD-IC-NF (1:1) and zein-THY $/ \gamma$-CD-IC-NF $(2: 1)$.

\subsection{Electrospinning}

Zein, zein-THY, zein-THY/ $\gamma$-CD-IC (1:1), and zein-THY $/ \gamma-C D-I C$ (2:1) solutions were loaded into a $5-\mathrm{mL}$ plastic syringe with metallic needle of $0.9 \mathrm{~mm}$ inner diameter. Then, the solutions were pumped at a constant rate $(0.5 \mathrm{~mL} / \mathrm{h})$ via syringe pump (KDS101; KD Scientific, Holliston, MA). A grounded metal covered by aluminum foil placed at a distance of $17 \mathrm{~cm}$ from the needle tip was used as a collector. A voltage of $17 \mathrm{kV}$ was applied from the high voltage power supply (AU Series; Matsusada Precision Inc., Osaka, Japan). All experiments were carried out in an enclosed Plexiglas box at $25^{\circ} \mathrm{C}$ and $18 \%$ relative humidity. The electrospun nanofibrous samples were kept at $4{ }^{\circ} \mathrm{C}$ till their analyses. 


\subsection{Characterizations and measurements}

The crystalline structure of powder of THY, $\gamma-\mathrm{CD}$, THY $/ \gamma$-CD-IC (1:1), THY $/ \gamma$-CD-IC (2:1), and zein-NF, zein-THY-NF, zein-THY $/ \gamma$ CD-IC-NF (1:1) and zein-THY/ $\gamma$-CD-IC-NF (2:1) was recorded via X-ray diffraction (XRD; X'Pert powder diffractometer; PANalytical, Almelo, The Netherlands) applying $\mathrm{Cu} \mathrm{K} \alpha$ radiation in a $2 \theta$ range $5-30^{\circ}$.

Thermal properties of THY, $\gamma$-CD, THY $/ \gamma$-CD-IC $(1: 1)$, THY $/ \gamma-C D-$ IC (2:1), zein-NF, zein-THY-NF, zein-THY/ $\gamma$-CD-IC-NF (1:1) and zein-THY $/ \gamma$-CD-IC-NF (2:1) were investigated by thermal gravimetric analysis (TGA; TA Q500; TA Instruments, New Castle, DE). The measurements were carried out under nitrogen atmosphere, and the samples were heated up to $500{ }^{\circ} \mathrm{C}$ at a constant heating rate of $20^{\circ} \mathrm{C} / \mathrm{min}$.

Proton nuclear magnetic resonance $\left({ }^{1} \mathrm{H}\right.$ NMR) spectra were recorded for THY $/ \gamma$-CD-IC $(1: 1)$ and THY $/ \gamma$-CD-IC $(2: 1)$ on a Bruker DPX-400 (Bruker Biospin, Rheinstetten, Germany). THY $/ \gamma$-CD-IC (1:1) and THY/ $\gamma$-CD-IC (2:1) were dissolved in d6-DMSO $(20 \mathrm{mg} /$ $\mathrm{mL}$ ) to determine the molar ratio of THY $/ \gamma$-CD-IC (1:1) and THY/ $\gamma$-CD-IC (2:1). Mestrenova software (Mestrelab Research) was used to integrate the chemical shifts $(\delta)$ given in parts per million (ppm).

A Physica MCR 301 rheometer (Anton Paar GmbH, Graz, Austria) was used to investigate the viscosity of zein, zein-THY, zein-THY $/ \gamma$-CD-IC (1:1), and zein-THY/ $\gamma$-CD-IC (2:1) solution at RT. The rheometer was equipped with a cone/plate accessory (spindle type CP 40-2) at a constant shear rate of $100 \mathrm{sec}^{-1}$. The conductivity of the solutions was determined by Inolab ${ }^{\circledR}$ Multi 720-WTW at RT.

The morphology of zein-NF, zein-THY-NF, zein-THY/ $\gamma$-CD-IC-NF (1:1) and zein-THY $/ \gamma$-CD-IC-NF (2:1) was examined via scanning electron microscopy (SEM, FEI-Quanta 200 FEG; FEI, Hillsboro, OR). For this purpose, samples were mounted on metal stubs using double-sided adhesive tape and coated with an $\mathrm{Au} / \mathrm{Pd}$ layer $(\sim 5 \mathrm{~nm})$ (PECS-682). About 100 fibers were analysed to calculate average fiber diameter (AFD) of nanofibers.

The amount of THY released from zein-THY-NF, zein-THY $/ \gamma-C D-$ IC-NF (1:1) and zein-THY/ $\gamma$-CD-IC-NF (2:1) was determined by headspace gas chromatography-mass spectrometry (HS GC-MS, Agilent Technologies 7890A gas chromatograph equipped with 5975C mass spectrometer; Agilent, Santa Clara, CA) for $5 \mathrm{~h}$. The capillary column was an HP-5MS (Agilent) $(30 \mathrm{~m} \times 0.25 \mathrm{~mm}$ i.d., $0.25 \mu \mathrm{m}$ film thickness). Nanofibers $(20 \mathrm{mg}$ ) were placed in 20 $\mathrm{mL}$ glass headspace vials and the vial was agitated at $500 \mathrm{rpm}$ and $37^{\circ} \mathrm{C}, 50^{\circ} \mathrm{C}$, and $75^{\circ} \mathrm{C}$. The oven temperature was initially held at $50{ }^{\circ} \mathrm{C}$ for $5 \mathrm{~min}$. Then, the temperature was raised with a gradient of $20^{\circ} \mathrm{C} / \mathrm{min}$ until $280^{\circ} \mathrm{C}$. The oven was held for $5 \mathrm{~min}$ at $280^{\circ} \mathrm{C}$. The instrument was operated with splitless injection and selected ion monitoring mode (SIM). NIST MS Search 2.0 library was used to identify the THY peaks. The release experiments were carried out from the three different parts of nanofibers in triplicate and the results are given as mean \pm standard deviation.

The antibacterial properties of the zein-THY-NF, zein-THY $/ \gamma$ CD-IC-NF (1:1) and zein-THY/ $\gamma$-CD-IC-NF (2:1) were investigated against Escherichia coli (E. coli, ATCC 10536) and Staphylococcus aureus (S. aureus, ATCC 25923) bacteria. Bacterial cells of E. coli (ATCC 10536) and S. aureus (ATCC 25923) were grown for $24 \mathrm{~h}$ on a shaker at $37{ }^{\circ} \mathrm{C}$ and $100 \mathrm{rpm}$. Inoculum was resuspended to provide a final density of $1 \times 10^{8}$ colony forming units $/ \mathrm{mL}(\mathrm{CFU} / \mathrm{mL})$ in phosphate buffered saline (PBS) according to 0.5 McFarland turbidity standard. UV sterilized ( $15 \mathrm{~min}, 1000 \mathrm{~J} \mathrm{~m}^{-2}$ ) nanofibers were then immersed in bacterial suspension in a $20-\mathrm{mL}$ conical tube, and the media were shaken at $37^{\circ} \mathrm{C}$ and $100 \mathrm{rpm}$ for $24 \mathrm{~h}$. The amounts of zein-THY-NF, zein-THY/ $\gamma$-CD-IC-NF (1:1) and zein-THY $/ \gamma$-CD-ICNF (2:1) used were $40 \mathrm{mg}, 53 \mathrm{mg}$, and $47 \mathrm{mg}$ (equivalent to $1.54 \mathrm{mg}$ of THY), respectively. Different dilutions $\left(10^{1}-10^{9}\right)$ were made by successively adding $1 \mathrm{~mL}$ culture into $9 \mathrm{~mL}$ of phosphate buffer solution. Then, $0.1 \mathrm{~mL}$ of the diluted culture were spread on a nutrient agar plate and incubated at $37^{\circ} \mathrm{C}$ overnight and $\mathrm{CFU} / \mathrm{mL}$ were counted.

The antibacterial capability of the mats was defined as follows:

Antibacterial activity $(\%)=100 \times(\mathrm{A}-\mathrm{B}) / \mathrm{A}$

where $A$ and $B$ are the number of colonies $(\mathrm{CFU} / \mathrm{mL}$ ) before and after nanofibers are added, respectively. The experiments were carried out from the three different parts of nanofibers in triplicate and the results are given as mean \pm standard deviation.

The antibacterial tests were also conducted after packing raw beef meat samples $(10 \mathrm{~g})$ with zein-THY-NF, zein-THY $/ \gamma$-CD-ICNF $(1: 1)$ and zein-THY/ $\gamma$-CD-IC-NF (2:1). Samples were prepared from fresh beef meat purchased from the local market by cutting it into squares. Firstly, meat samples $(10 \mathrm{~g})$ were packed with nanofibers; then, they were put in polyethylene zip bags and stored at $4{ }^{\circ} \mathrm{C}$ for 5 days. Packing of meat samples with nanofibers was done by putting them in the centre of the nanofiber, thus nanofibers covered all surfaces of the meat sample. The amounts of zein-THY-NF, zein-THY/ $\gamma$-CD-IC-NF (1:1) and zein-THY/ $\gamma$-CDIC-NF (2:1) used for packing were $40 \mathrm{mg}$, $53 \mathrm{mg}$, and $47 \mathrm{mg}$ (equivalent to $1.54 \mathrm{mg}$ of THY), respectively. The unpacked meat was served as blank control. At the end of 1, 2, and 5 days of storage, nanofibers were removed and meat samples ( $10 \mathrm{~g})$ were combined with sterile PBS and vortexed for 2 min. Appropriate dilutions in PBS were plated in triplicate on plate count agar for determination of aerobes in the samples. Only microbial colonies from the surface were collected into the PBS. Colonies were counted after incubation of the plates at $35{ }^{\circ} \mathrm{C}$ for $24-48 \mathrm{~h}$. The microorganism counts were expressed in $\log \mathrm{CFU} / \mathrm{g}$ of meat sample. The experiments were performed from the three different parts of nanofibers in triplicate and the results are given as mean \pm standard deviation.

\subsection{Computational method}

The first-principles calculations based on density functional theory (DFT) (Hohenberg \& Walter, 1964; Kohn \& Sham, 1965) were performed by using the Vienna Ab initio simulation package (Kresse \& Furthmuller, 1996a, 1996b). The exchange-correlation was described by the Perdew-Burke-Ernzerhof functional of generalized gradient approximation (GGA-PBE) (Perdew et al., 1992). The Van der Waals correction was taken into account to better characterize the intermolecular interactions (Grimme, 2006). The projector augmented-wave method (PAW) (Blochl, 1994) was utilized for pseudopotentials of all elements with a plane-wave basis set having a kinetic energy cut off of $520 \mathrm{eV}$. Calculations were made for $1: 1$ and 2:1 stoichiometry of THY $/ \gamma-C D-I C s$ in vacuum and solvent (water). The initial structures of THY and $\gamma$-CD molecules were obtained from Cambridge Structural Database (Allen, 2002). All the structures including THY $/ \gamma$-CD-ICs were optimized by using the conjugate gradient algorithm without any constraints, by setting convergence criteria on the total energy and force to $10^{-4} \mathrm{eV}$ and $10^{-2} \mathrm{eV} / \AA$, respectively. The effect of water on the formation of THY $/ \gamma$-CD-ICs has been elucidated by using implicit solvent model (Fattebert \& Gygi, 2003), which includes dispersive interactions. This model splits the system into an explicit part (solute), which is treated quantum mechanically and an implicit part (solvent) which is treated as a continuum (Andreussi, Dabo, \& Marzari, 2012). The intermolecular interactions between water molecules are gathered from continuum dielectric description of the solvent (Mathew, Sundararaman, Letchworth-Weaver, Arias, \& Hennig, 2014; Petrosyan, Rigos, \& Arias, 2005). Moreover, the solvation energies of THY, THY $/ \gamma$-CD-IC $(1: 1)$ and THY $/ \gamma-C D-I C$ $(2: 1)$ in water was calculated as well. 


\subsection{Statistical analyses}

Statistical analyses were performed using GraphPad Prism 5. To determine the statistical difference for both release and antibacterial activity tests, one-way ANOVA and Tukey's multiple comparison post-tests $(p<0.05)$ were employed.

\section{Results and discussion}

\subsection{Molecular modelling of $T H Y / \gamma-C D-I C$}

Initially, molecular modelling studies were performed to discover if inclusion complexation between guest molecules (i.e. THY) and the host molecules (i.e. $\gamma$-CD) occurs and if that is the case, what would be the molar ratio for THY $/ \gamma$-CD-IC. Inclusion complexation formation is strongly correlated with the interactions between guest and host molecules and the environment. These interactions can be altered by using different type of solvents leading to strong/weak complexation. The accurate molecular models based on ab initio calculations can give insight into key points of these reactions. Therefore, to interpret the experimental data, structural optimizations of THY molecule, THY dimer, $\gamma-\mathrm{CD}$, and their ICs were carried out in vacuum, followed by optimizations in water as a solvent environment. In addition to $1: 1$ stoichiometry of THY $/ \gamma-C D-$ IC, $2: 1$ stoichiometry of THY $/ \gamma-\mathrm{CD}$-IC considering the size of THY and the cavity of $\gamma$-CD molecules were modelled. THY dimer has been formed by orienting phenol parts to each other at an optimum distance of 3.7 Å. To form IC, single THY molecule (1:1) or dimer (2:1) approach the cavity of $\gamma$-CD step-by-step for possible orientations of THY. The most favourable geometry is determined by calculating the total energy of the system at each step after the structural optimization. Once the lowest energy configuration is obtained, the complexation energy ( $\left.\mathrm{E}_{\text {comp }}\right)$ is calculated as

$\mathrm{E}_{\text {comp }}=\mathrm{E}_{\mathrm{CD}}+\mathrm{E}_{\text {guest }}-\mathrm{E}_{\mathrm{IC}}$

where $E_{C D}, E_{\text {guest }}$, and $E_{I C}$ are the total energy (in vacuum or solvent) of $\gamma-C D$; THY molecule or dimer; and IC for $1: 1$ or $2: 1$ stoichiometry, respectively. $E_{\text {comp }}$ in vacuum and water together with solvation energy ( $\left.E_{\text {solv }}\right)$ for $1: 1$ and 2:1 stoichiometry are summarized in Table S2.

The configuration in which two methyl groups of THY molecule or dimer heading towards the cavity of $\gamma$-CD is preferred; thus the phenol group of THY is facing outwards of the narrow rim (Fig. 2ad). In the study of Mulinacci et al., influence of water during the complexation process was evaluated by inserting a new parameter, dielectric constant (D) in the calculations. When experimental and modelling studies were considered together, the results showed that the conformation in which isopropyl group of THY lay closer to the wider rim of the $\beta-C D$ is more favourable (Mulinacci, Melani, Vincieri, Mazzi, \& Romani, 1996). Even though the CD type is different in this study, the favourable configuration determined based on the calculations is similar to our results. According to $\mathrm{E}_{\text {comp }}$ calculated in both vacuum and solvent, THY $/ \gamma$-CD-IC $(2: 1)$ stoichiometry is much more favourable than THY/ $\gamma$-CD-IC (1:1) stoichiometry. Even though THY: $\gamma-C D-I C$ can be formed in both vacuum and solvent medium, $\mathrm{E}_{\text {comp }}$ is slightly higher in water compared to vacuum. Water increases the complexation energy in all cases, due to the hydrophobic nature of the THY and $\gamma$-CD cavity. Additionally, the polar phenol part of THY favours outward orientation in polar solvent medium for $1: 1$ and 2:1 stoichiometry. $\mathrm{E}_{\mathrm{solv}}$ of THY $/ \gamma-\mathrm{CD}-\mathrm{ICs}$ in water is calculated as

$\mathrm{E}_{\text {solv }}=\mathrm{E}(\mathrm{IC})_{(\text {solvent })}-\mathrm{E}(\mathrm{IC})_{(\text {vacuum })}$

where $\mathrm{E}(\mathrm{IC})_{\text {(vacuum) }}$ and $\mathrm{E}(\mathrm{IC})_{\text {(solvent) }}$ is the total energy of THY $/ \gamma-\mathrm{CD}$ IC in vacuum and solvent (water), respectively. The calculated $\mathrm{E}_{\text {solv }}$
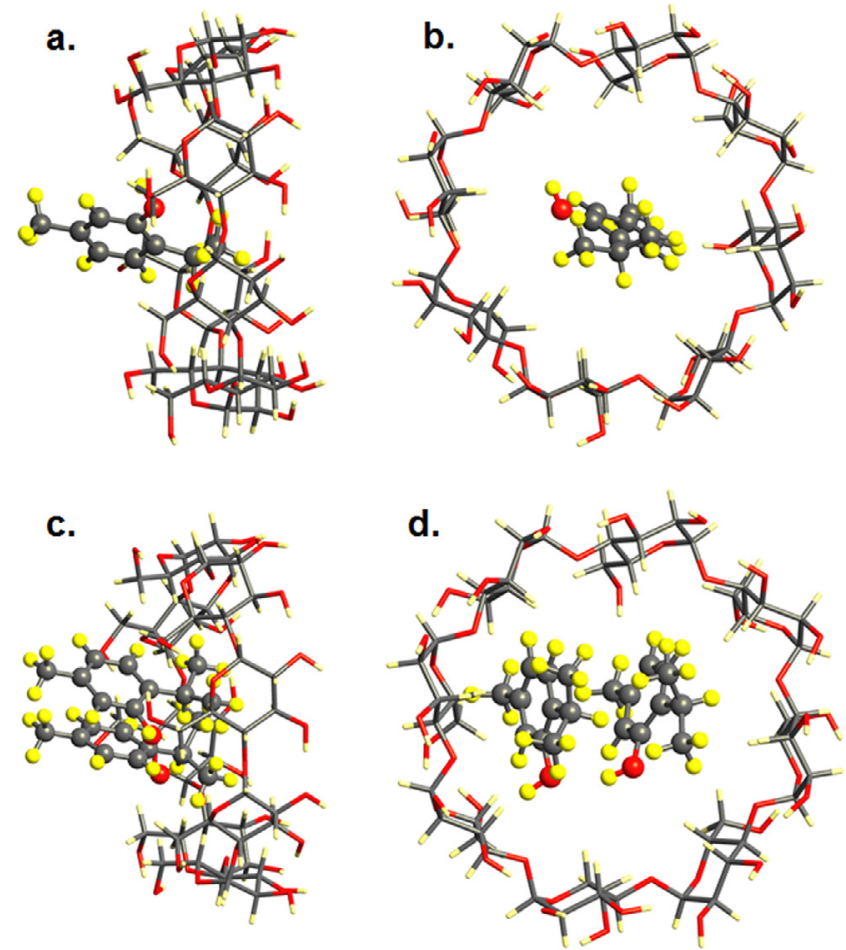

Fig. 2. Side and top view of THY $/ \gamma$-CD-IC for (a)-(b) 1:1 and (c)-(d) 2:1 stoichiometry. Gray, red, and yellow spheres represent carbon, oxygen, and hydrogen atoms, respectively. (For interpretation of the references to colour in this figure legend, the reader is referred to the web version of this article.)

of THY $/ \gamma$-CD-IC (1:1) and THY $/ \gamma$-CD-IC (2:1) are $-83 \mathrm{kcal} / \mathrm{mol}$ and $-74 \mathrm{kcal} / \mathrm{mol}$, respectively. Although these values do not reveal the absolute solubility of ICs, they indicate high solubility trend of $\mathrm{THY} / \gamma$-CD-ICs in water for both cases when compared to cyclodextrin-free pristine THY $(-4.6 \mathrm{kcal} / \mathrm{mol})$.

\subsection{The molar ratio of $T H Y / \gamma-C D-I C$}

For the preparation of inclusion complexation between THY and $\gamma$-CD (THY $/ \gamma$-CD-IC), the initial molar ratio of $1: 1$ and $2: 1$ for THY: $\gamma$-CD was used based on the modelling studies but the final molar ratio after complex formation is important, since these precipitated crystalline aggregates of THY $/ \gamma$-CD-IC were directly incorporated in zein solutions for the electrospinning of nanofibers. Proton nuclear magnetic resonance $\left({ }^{1} \mathrm{H}\right.$ NMR) is a useful technique to determine the molar ratio of inclusion complexes by dissolving the precipitated $\mathrm{CD}$-IC crystals in a common deuterated solvent, which is DMSO- $d_{6}$ in the case of THY $/ \gamma-C D-I C$. The ${ }^{1} \mathrm{H}$ NMR spectra for THY $/ \gamma$-CD-IC (1:1) and THY/ $\gamma$-CD-IC (2:1) are given in Fig. S1 and the molar ratio of THY $/ \gamma$-CD-IC (1:1) and THY $/ \gamma$-CD-IC $(2: 1)$ was calculated by taking the integration of the peak ratio of the characteristic chemical shifts of THY (9.1 ppm) and $\gamma-C D$ (4.5 ppm). As a result, the molar ratio of THY $/ \gamma$-CD-IC $(1: 1)$ and $\mathrm{THY} / \gamma$-CD-IC $(2: 1)$ was calculated as $\sim 1: 1$ and $\sim 2: 1$, respectively. Briefly, it was revealed from the ${ }^{1} \mathrm{H}$ NMR study that the initial molar ratio of $\mathrm{THY} / \gamma-\mathrm{CD}-\mathrm{IC}$ in water was totally preserved for the precipitated $\mathrm{THY} / \gamma-\mathrm{CD}$-IC crystalline samples. The studies in the literature are usually on the complexation of THY with $\beta-C D$ and its derivatives. In the study of Bethanis et al., THY was included into $\beta$-CD at $1: 1,1.1 .5$, and $1: 2$ molar ratio and crystals of the best quality were obtained from aqueous solutions of $1: 1$ molar ratio for $\beta-C D$ inclusion compounds with THY (Bethanis et al., 2013). 
In a study conducted by Kfoury et al., THY was inserted into $\alpha-C D$, $\beta-C D, \gamma-C D, H P \beta C D, R A M E B$, and CRYSMEB at 1:1 molar ratio, but the molar ratio of $\gamma-C D$ with thymol was not calculated after the synthesis of CD-ICs (Kfoury et al., 2016).

\subsection{Thermal analyses of $T H Y / \gamma-C D-I C$}

The thermal stability of THY, $\gamma-\mathrm{CD}$, THY $/ \gamma-\mathrm{CD}-\mathrm{IC}(1: 1)$, and THY/ $\gamma$-CD-IC (2:1) were investigated by thermal gravimetric analysis (TGA) (Fig. S2a). The thermal evaporation of THY took place between $50{ }^{\circ} \mathrm{C}$ and $145^{\circ} \mathrm{C}$ with a main peak point at $130{ }^{\circ} \mathrm{C} . \gamma$ $\mathrm{CD}$ exhibited two weight losses below $100^{\circ} \mathrm{C}$ and above $275^{\circ} \mathrm{C}$ which belong to the water loss from the cavity and main thermal degradation of $\mathrm{CD}$, respectively. THY $/ \gamma$-CD-IC $(1: 1)$ and THY $/ \gamma$ CD-IC (2:1) lost weight in three steps. The first loss, which occurred below $100^{\circ} \mathrm{C}$, was the loss of water molecules in the cavity of CDs. The second weight loss that belongs to thermal evaporation of THY observed in THY $/ \gamma$-CD-IC $(1: 1)$ and THY $/ \gamma$-CD-IC (2:1) was between $125-280^{\circ} \mathrm{C}$ and $115-280^{\circ} \mathrm{C}$, respectively. Therefore, the apparent increment in the thermal stability of THY suggested the formation of true complexation between THY and $\gamma$-CD in both samples. The shifting of thermal evaporation of geraniol, which is a volatile compound, to a higher temperature was observed in a previously published study and this was the indication of true complexation between the guest and CDs (Aytac et al., 2016). The last weight loss, which occurred at higher temperatures than $275^{\circ} \mathrm{C}$, corresponded to the main thermal degradation of $\gamma-\mathrm{CD}$. The theoretical amount of THY in THY $/ \gamma$-CD-IC (1:1) and THY $/ \gamma$ CD-IC $(2: 1)$ was $10.7 \%(\mathrm{w} / \mathrm{w})$ and $18.7 \%(\mathrm{w} / \mathrm{w})$, respectively. The calculated amount of THY in THY $/ \gamma$-CD-IC $(1: 1)$ and THY $/ \gamma$-CD-IC $(2: 1)$ was $\sim 9.6 \%(\mathrm{w} / \mathrm{w})$ and $18.6 \%(\mathrm{w} / \mathrm{w})$, respectively. According to TGA data, precipitated crystal samples of THY/ $\gamma$-CD-IC (1:1) and THY $/ \gamma$-CD-IC $(2: 1)$ have preserved $89.7 \%$ and $99.5 \%$ of the initial amount of THY, respectively. Although, it is difficult to calculate the exact molar ratio of THY: $\gamma-\mathrm{CD}$ by TGA, ${ }^{1} \mathrm{H}$ NMR data and TGA data correlate with each other and it is evident that the initial molar ratio of $1: 1$ and $2: 1$ for THY: $\gamma-C D$ was preserved to a great extent for both of the precipitated THY $/ \gamma-\mathrm{CD}$-IC samples. TGA of zein-NF, zein-THY-NF, zein-THY/ $\gamma$-CD-IC-NF (1:1) and zein-THY/ $\gamma$-CD-IC-NF (2:1) was investigated as well and thermograms are given in Fig. S2b. The shifting of thermal evaporation of THY to higher temperatures was seen in zein-THY/ $\gamma$-CD-IC-NF $(1: 1)$ and zein-THY $/ \gamma$-CD-IC-NF (2:1), as compared with zein-THY-NF. This result supports the formation of true complexes between THY and $\gamma$-CD in zein-THY $/ \gamma$-CD-IC-NF $(1: 1)$ and zein-THY/ $\gamma$-CDIC-NF (2:1).

\subsection{Crystalline structure of $T H Y / \gamma-C D-I C$ and nanofibers}

$\mathrm{X}$-ray diffraction (XRD) was performed for thymol (THY), $\gamma-\mathrm{CD}$, THY $/ \gamma$-CD-IC (1:1), and THY $/ \gamma$-CD-IC $(2: 1)$ and the diffraction patterns are displayed in Fig. S3a. Intense and sharp diffraction peaks of THY were observed, showing its crystalline structure. In the case of THY $/ \gamma$-CD-IC $(1: 1)$ and THY $/ \gamma$-CD-IC (2:1), the absence of the crystalline peaks of THY is the proof of complex formation between THY and $\gamma-C D$, since the guest molecules are separated from each other during CD-IC formation (Giordano, Novak, \& Moyano, 2001). In addition to that, transformation of crystalline structure of as-received $\gamma$-CD from cage packing to tetragonal channel-type packing with a major peak at $2 \theta$ value of $7.5^{\circ}$ and minor peaks at $2 \theta$ values of $14^{\circ}, 15^{\circ}, 16^{\circ}, 17^{\circ}$ and $22^{\circ}$ is a strong proof of complex formation between THY and $\gamma-\mathrm{CD}$. This result is in agreement with the study of Kayaci et al., in which vanillin was included into the $\gamma$-CD cavity and major and minor peaks of tetragonal channeltype packing of $\gamma$-CD was observed in addition to disappearance of the crystalline peaks of vanillin (Kayaci \& Uyar, 2012). XRD patterns of zein nanofibers (zein-NF), THY encapsulated zein nanofibers without CD (zein-THY-NF), THY $/ \gamma$-CD $(1: 1)$ encapsulated zein nanofibers (zein-THY $/ \gamma$-CD-IC-NF $(1: 1)$ ) and THY $/ \gamma$-CD $(2: 1)$ encapsulated zein nanofibers (zein-THY $/ \gamma$-CD-IC-NF $(2: 1)$ ) are given in Fig. S3b. The zein-THY $/ \gamma$-CD-IC-NF $(1: 1)$ and zein-THY/ $\gamma$-CD-IC-NF (2:1) exhibited a broad XRD pattern without the sharp crystalline peaks of $\mathrm{THY} / \gamma-\mathrm{CD}-\mathrm{IC}$, possibly due to the amorphous distribution of THY $/ \gamma-\mathrm{CD}-\mathrm{IC}$ in the fiber matrix. The similar situation in which CD-ICs were distributed amorphously was observed when quercetin, a crystalline molecule, was included into $\beta-C D$ and then incorporated into electrospun nanofibers (Aytac et al., 2016b). Similarly, THY in the zein-THY-NF sample was also distributed in the zein fiber matrix without any crystal formation. In short, XRD revealed that both THY $/ \gamma$-CD-IC $(1: 1$ and $2: 1)$ and THY were distributed in the electrospun zein fiber matrix without forming any considerable crystal aggregates.

\subsection{Morphology analyses of nanofibers}

The morphological characterization of zein-NF, zein-THY-NF, zein-THY $/ \gamma$-CD-IC-NF (1:1) and zein-THY/ $\gamma$-CD-IC-NF (2:1) was carried out via scanning electron microscopy (SEM). SEM images of the nanofibers are depicted in Fig. 3a-d. The electrospinning of bead-free and uniform nanofibers from zein, zein-THY and zein$\mathrm{THY} / \gamma$-CD-IC solutions was achieved by optimizing electrospinning parameters. The average fiber diameter (AFD) was determined as $155 \pm 30 \mathrm{~nm}, 205 \pm 50 \mathrm{~nm}, 245 \pm 70 \mathrm{~nm}$, and $415 \pm 100 \mathrm{~nm}$ for zein-NF, zein-THY-NF, zein-THY/ $\gamma$-CD-IC-NF (1:1) and zein-THY/ $\gamma$-CD-IC-NF (2:1), respectively. AFD difference of nanofibers is possibly related with the viscosity and conductivity of the solutions. The viscosity of zein-THY solution is higher; whereas the conductivity is almost the same with zein solution; therefore, zein-THY-NF has higher diameter compared to zein-NF. AFD of zein-THY $/ \gamma$-CD-IC-NF (1:1) and zein-THY $/ \gamma$-CD-IC-NF (2:1) are higher than zein-NF and zein-THY-NF, due to the higher viscosity and lower conductivity of these solutions.

\subsection{Release study}

The amount of THY released from zein-THY-NF, zein-THY $/ \gamma-\mathrm{CD}-$ IC-NF (1:1), and zein-THY/ $\gamma$-CD-IC-NF $(2: 1)$ at $37^{\circ} \mathrm{C}, 50{ }^{\circ} \mathrm{C}$, and $75^{\circ} \mathrm{C}$ for $5 \mathrm{~h}$ are shown in Fig. $4 \mathrm{a}-\mathrm{c}$. The release of THY from the nanofibers was increased gradually with time and became constant in the last stage. The released amount of THY was more from

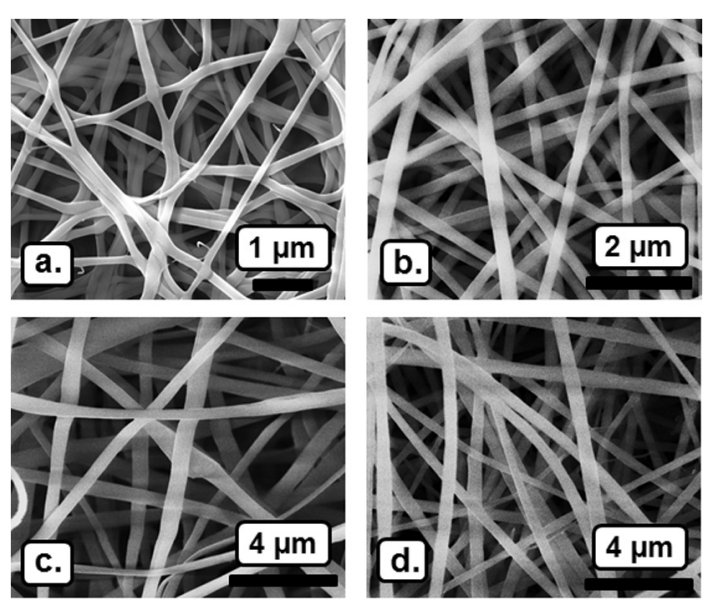

Fig. 3. SEM images of electrospun nanofibers obtained from solutions of (a) zein, (b) zein-THY, (c) zein-THY/ $\gamma$-CD-IC (1:1), and (d) zein-THY/ $\gamma$-CD-IC (2:1). 
a.

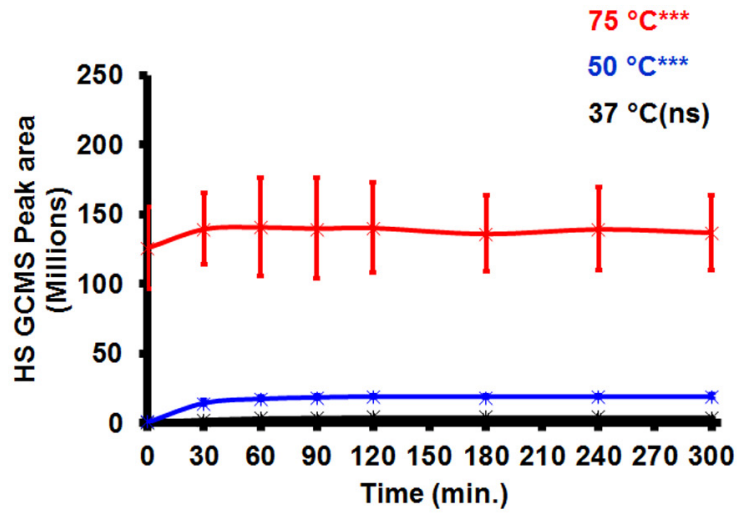

b.
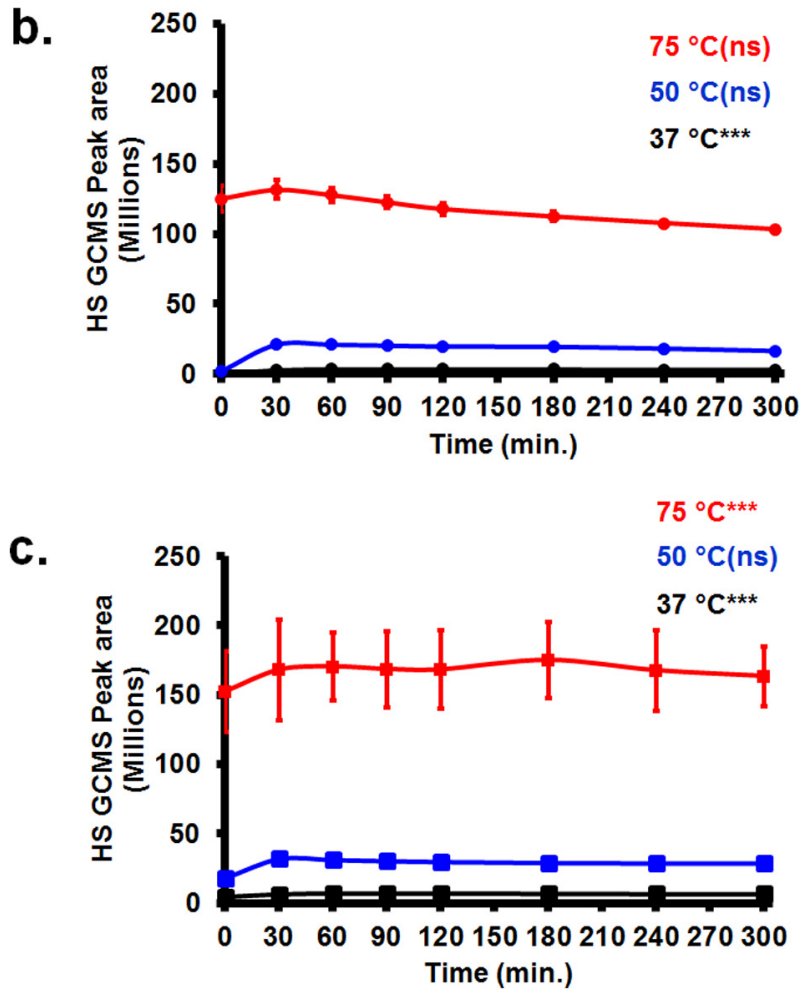

Fig. 4. The cumulative release of THY from (a) zein-THY-NF, (b) zein-THY/ $\gamma$-CD-ICNF (1:1), and (c) zein-THY $/ \gamma$-CD-IC-NF $(2: 1)$ at $37^{\circ} \mathrm{C}, 50{ }^{\circ} \mathrm{C}$, and $75^{\circ} \mathrm{C}(n=3)$. The error bars in the figure represent the standard deviation (SD). (ns: not significant, ${ }^{*}$ : $\left.p \leq 0.05,{ }^{* *}: p \leq 0.01,{ }^{* * *}: p \leq 0.001\right)$.

zein-THY $/ \gamma$-CD-IC-NF (2:1) compared to zein-THY-NF and zein$\mathrm{THY} / \gamma$-CD-IC-NF (1:1). The reason for the less amount of release from zein-THY-NF compared to zein-THY/ $\gamma$-CD-IC-NF (2:1) could be the absence of CD-IC that was preserving THY from evaporation. In the case of zein-THY/ $\gamma$-CD-IC-NF (1:1), the stability and preservation rate of THY was lower than the complex in zein-THY $/ \gamma-C D-$ IC-NF (2:1) as observed in computational modelling and TGA data. So, the zein-THY $/ \gamma$-CD-IC-NF $(1: 1)$ could not preserve THY as effectively as zein-THY $/ \gamma$-CD-IC-NF (2:1). In addition, as the temperature increased, the amount of THY released increased as well for all nanofibrous web samples, due to the increment in the motion of the polymer chains (Galotto, Torres, Guarda, Moraga, \& Romero, 2011) and enhanced kinetic energy of THY causing hydrogen bonds to weaken (Caka et al., 2016). To our knowledge, there is no study in the literature, in which THY/CD-ICs were incorporated into electrospun nanofibers. The relative humidity $(\mathrm{RH})$ dependent release of THY from THY/ $\beta$-CD-ICs was investigated by Cevallos et al., and it was deduced that release was not observed up to $84 \%$ (at RT for 70 days). However, when the RH increased to $84 \%$ (at RT) the release of THY was very low up to 70 days and after that point it increased slightly. If the RH increased to $97 \%$, the release was higher, as compared to the same time period at $84 \% \mathrm{RH}$, up to 25 days of storage, after then the release increased almost linearly with storage time up to 70 days (Cevallos et al., 2010). The morphology and AFD of nanofibers after the release experiment was investigated by SEM. As seen from the representative SEM images given in Fig. S4, zein-THY/ $\gamma$-CD-IC-NF (2:1) kept its fibrous structure after the release experiment at $50^{\circ} \mathrm{C}$ and $75^{\circ} \mathrm{C}$ for $5 \mathrm{~h}$. In addition, AFD of zein-THY/ $\gamma$-CD-IC-NF (2:1) was calculated to be $465 \pm 75 \mathrm{~nm}$ and $435 \pm 95 \mathrm{~nm}$ after the release experiment at $50{ }^{\circ} \mathrm{C}$ and $75^{\circ} \mathrm{C}$, respectively. Therefore, it was concluded that there is no significant difference in AFD of nanofibers before $(415 \pm 100 \mathrm{~nm})$ and after the release experiment at $50^{\circ} \mathrm{C}$ and $75^{\circ} \mathrm{C}$.

\subsection{Antibacterial activity}

It is well-known that THY, one of the major components of thyme oil, has useful antibacterial activity against various microorganisms (Bozin, Mimica-Dukic, Simin, \& Anackov, 2006). The antibacterial activities of zein-THY-NF, zein-THY $/ \gamma-C D-I C-N F$ $(1: 1)$, and zein-THY/ $\gamma$-CD-IC-NF (2:1) were tested against model Gram-negative E. coli and Gram-positive S. aureus) bacteria via colony counting method (Fig. 5). The antibacterial activity (\%) of zeinTHY $/ \gamma$-CD-IC-NF $(1: 1)$ and zein-THY $/ \gamma$-CD-IC-NF (2:1) was more than zein-THY-NF against both $E$. coli and $S$. aureus. Zein-THY $/ \gamma-$ CD-IC-NF (2:1) exhibited better antibacterial activity than zeinTHY $/ \gamma$-CD-IC-NF (1:1) against $E$. coli, owing to the higher stability, preservation, and release of THY as shown in computational modelling, TGA, and HS GC-MS studies, respectively. Moreover, higher antibacterial activity of nanofibers for $S$. aureus than $E$. coli might be related with the cell wall composition of Gram-negative and Gram-positive bacteria. Thus, E. coli has a thin peptidoglycan layer and an outer layer of lipoproteins, lipopolysaccharides and phospholipids, while the cell wall of $S$. aureus comprises a peptidoglycan layer with lots of pores. The porous cell wall structure of $S$. aureus makes it easy to be permeated by THY (Madigan, Clark, Stahl, \& Martinko, 2010).

Zein-THY-NF, zein-THY/ $\gamma$-CD-IC-NF (1:1), and zein-THY/ $\gamma$-CDIC-NF $(2: 1)$ were packed on meat samples to show the applicability of these nanofibrous webs for antibacterial food packaging. The number of bacterial colonies (CFU/g) counted in meat samples packed with nanofibers during 5 days of storage at $4{ }^{\circ} \mathrm{C}$ are given in Table 1. The initial amount of bacterial colonies of the control (meat) was $16.1 \pm 0.2 \times 10^{2} \mathrm{CFU} / \mathrm{g}$ on the first day, and it increased up to $97.6 \pm 0.5 \times 10^{7} \mathrm{CFU} / \mathrm{g}$ on the fifth day. Since food is considered as spoiled when the amount of bacteria exceeds $10^{7} \mathrm{CFU} / \mathrm{g}$; the experiment was stopped on the fifth day (Wen, Zhu, Feng et al., 2016). The number of bacteria counted in meat samples packed with nanofibrous webs was less than the control sample on the first, second, and fifth days of the storage. So, it was deduced that nanofibrous webs encapsulating THY prolong the shelf life of the meat. Moreover, the lowest amount of bacteria was observed in zein-THY $/ \gamma$-CD-IC-NF (2:1), and this result is correlated well with the release and antibacterial activity results. Tao et al. showed the antimicrobial activity of THY/ $\beta-C D$-ICs against $E$. coli was higher than free THY. The effect of THY/ $\beta$-CD-ICs on the shelf life of pork meat has been previously investigated and similar results were obtained (Tao et al., 2014). However we believe that the nanofibers are easier to use than the powdered CD-ICs for packaging applications. 


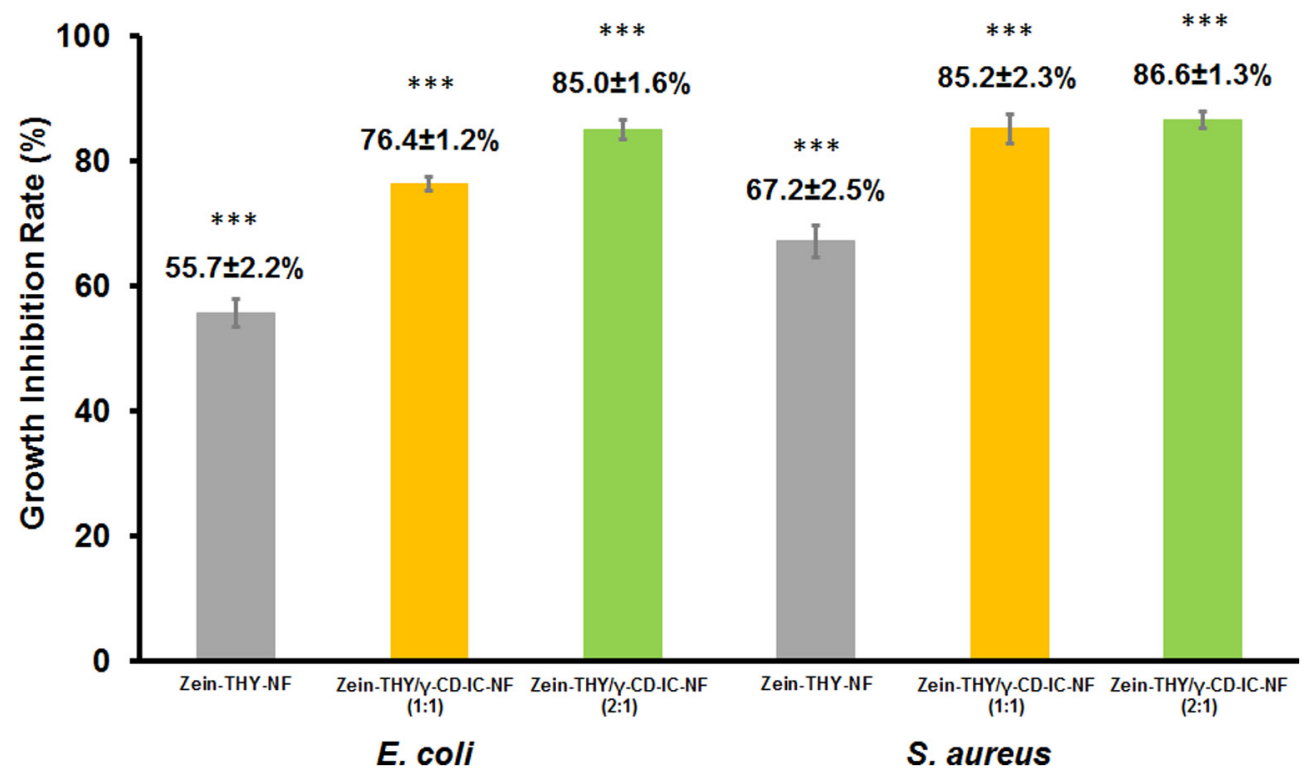

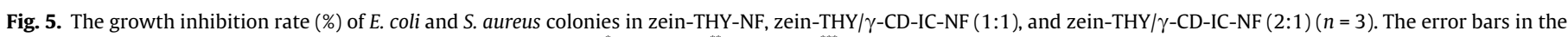
figure represent the standard deviation (SD). (ns: not significant, ${ }^{*}: p \leq 0.05,{ }^{* *}: p \leq 0.01,{ }^{* * *}: p \leq 0.001$ ).

Table 1

The number of bacterial colonies ( $\mathrm{CFU} / \mathrm{g}$ ) counted in meat samples packed with nanofibers during 5 days of storage at $4{ }^{\circ} \mathrm{C}\left({ }^{* * *}: p \leq 0.001\right)$.

\begin{tabular}{|c|c|c|c|}
\hline Samples & Day $1^{* * *}$ & Day $2^{* * *}$ & Day $5^{* * *}$ \\
\hline Control (meat) & $16.1 \pm 0.2 \times 10^{2}$ & $25.7 \pm 0.5 \times 10^{4}$ & $97.6 \pm 0.5 \times 10^{7}$ \\
\hline Zein/THY-NF & $7.2 \pm 0.3 \times 10^{2}$ & $54.9 \pm 0.4 \times 10^{3}$ & $55.5 \pm 0.4 \times 10^{6}$ \\
\hline $\begin{array}{l}\text { Zein/THY } / \gamma-C D-I C-N F \\
\quad(1: 1)\end{array}$ & $9.1 \pm 0.5 \times 10^{2}$ & $61.8 \pm 0.2 \times 10^{3}$ & $24.1 \pm 0.9 \times 10^{6}$ \\
\hline $\begin{array}{l}\text { Zein } / \text { THY } / \gamma \text {-CD-IC-NF } \\
\quad(2: 1)\end{array}$ & $5.7 \pm 0.9 \times 10^{2}$ & $46.7 \pm 0.7 \times 10^{3}$ & $11.2 \pm 0.3 \times 10^{6}$ \\
\hline
\end{tabular}

\section{Conclusion}

Electrospun nanofibers were produced from THY $/ \gamma$-CD-IC $(1: 1)$ and THY $/ \gamma$-CD-IC (2:1) incorporated zein solution via electrospinning technique. SEM images revealed the bead-free and uniform nanofibers were successfully electrospun. Inclusion complexation was investigated by experimental (XRD, TGA, and ${ }^{1} \mathrm{H}$ NMR) and computational modelling studies. The results suggested the successful formation of complex between THY and $\gamma$-CD at $1: 1$ and $2: 1$ molar ratio. However, it is worth mentioning that THY $/ \gamma-C D-$ IC $(2: 1)$ possessed higher preservation rate and stability as compared to THY $/ \gamma$-CD-IC $(1: 1)$. The release of THY was higher from zein-THY $/ \gamma$-CD-IC-NF (2:1) compared to other nanofibers, due to the higher stability and preservation rate of THY in THY $/ \gamma-C D-I C$ $(2: 1)$. Similarly, zein-THY $/ \gamma$-CD-IC-NF $(2: 1)$ had stronger antibacterial activity than zein-THY-NF and zein-THY $/ \gamma$-CD-IC-NF (1:1) against $E$. coli and $S$. aureus. In brief, zein-THY/ $\gamma-C D-I C-N F$ nanofibrous webs were most effective at reducing the bacterial count in meat stored up to 5 days at $4{ }^{\circ} \mathrm{C}$. Thus, these webs show potential as an antibacterial food packaging material.

\section{Acknowledgement}

Dr. Uyar acknowledges The Scientific and Technological Research Council of Turkey (TUBITAK)-Turkey (Project \# $111 \mathrm{M} 459)$ for funding this research. Dr. Uyar and Dr. Durgun acknowledge The Turkish Academy of Sciences - Outstanding Young Scientists Award Program (TUBA-GEBIP) for partial funding of the research. Z. Aytac thanks to TUBITAK-BIDEB and TUBITAK (project \# 111M459 and 213M185) for the PhD scholarship.

\section{Appendix A. Supplementary data}

Supplementary data associated with this article can be found, in the online version, at http://dx.doi.org/10.1016/j.foodchem.2017. 04.095.

\section{References}

Allen, F. H. (2002). The Cambridge Structural Database: A quarter of a million crystal structures and rising. Acta Crystallographica Section B: Structural Science, 58(3), 380-388.

Andreussi, O., Dabo, I., \& Marzari, N. (2012). Revised self-consistent continuum solvation in electronic-structure calculations. The Journal of Chemical Physics, 136(6), 064102.

Aytac, Z., Dogan, S. Y., Tekinay, T., \& Uyar, T. (2014). Release and antibacterial activity of allyl isothiocyanate/ $\beta$-cyclodextrin complex encapsulated in electrospun nanofibers. Colloids and Surfaces B: Biointerfaces, 120, 125-131.

Aytac, Z., Kusku, S. I., Durgun, E., \& Uyar, T. (2016a). Encapsulation of gallic acid/cyclodextrin inclusion complex in electrospun polylactic acid nanofibers: Release behavior and antioxidant activity of gallic acid. Materials Science and Engineering: C, 63, 231-239.

Aytac, Z., Kusku, S. I., Durgun, E., \& Uyar, T. (2016b). Quercetin/ $\beta$-cyclodextrin inclusion complex embedded nanofibres: Slow release and high solubility. Food Chemistry, 197, 864-871.

Aytac, Z., Sen, H. S., Durgun, E., \& Uyar, T. (2015). Sulfisoxazole/cyclodextrin inclusion complex incorporated in electrospun hydroxypropyl cellulose nanofibers as drug delivery system. Colloids and Surfaces B: Biointerfaces, 128, 331-338.

Aytac, Z., \& Uyar, T. (2016). Antioxidant activity and photostability of $\alpha$-tocopherol/ $\beta$-cyclodextrin inclusion complex encapsulated electrospun polycaprolactone nanofibers. European Polymer Journal, 79, 140-149.

Aytac, Z., Yildiz, Z. I., Kayaci-Senirmak, F., San Keskin, N. O., Tekinay, T., \& Uyar, T. (2016). Electrospinning of polymer-free cyclodextrin/geraniol-inclusion complex nanofibers: enhanced shelf-life of geraniol with antibacterial and antioxidant properties. RSC Advances, 6(52), 46089-46099.

Bethanis, K., Tzamalis, P., Tsorteki, F., Kokkinou, A., Christoforides, E., \& Mentzafos, D. (2013). Structural study of the inclusion compounds of thymol, carvacrol and eugenol in $\beta$-cyclodextrin by X-ray crystallography. Journal of Inclusion Phenomena and Macrocyclic Chemistry, 77(1-4), 163-173.

Blochl, P. E. (1994). Projector augmented-wave method. Physical Review B, 50(24), 17953.

Bozin, B., Mimica-Dukic, N., Simin, N., \& Anackov, G. (2006). Characterization of the volatile composition of essential oils of some Lamiaceae spices and the antimicrobial and antioxidant activities of the entire oils. Journal of Agricultural and Food Chemistry, 54, 1822-1828.

Caka, M., Turkcan, C., Aktas Uygun, D., Uygun, M., Akgol, S., \& Denizli, A. (2016). Controlled release of curcumin from poly (HEMA-MAPA) membrane. Artificial Cells, Nanomedicine, and Biotechnology, 1-6.

Cevallos, P. A. P., Buera, M. P., \& Elizalde, B. E. (2010). Encapsulation of cinnamon and thyme essential oils components (cinnamaldehyde and thymol) in $\beta$ - 
cyclodextrin: effect of interactions with water on complex stability. Journal of Food Engineering, 99(1), 70-75.

Chen, G., \& Liu, B. (2016). Cellulose sulfate based film with slow-release antimicrobial properties prepared by incorporation of mustard essential oil and $\beta$-cyclodextrin. Food Hydrocolloids, 55, 100-107.

Del Valle, E. M. (2004). Cyclodextrins and their uses: a review. Process Biochemistry, 39(9), 1033-1046.

Fattebert, J. L., \& Gygi, F. (2003). First-principles molecular dynamics simulations in a continuum solvent. International Journal of Quantum Chemistry, 93(2), 139-147.

Galotto, M. J., Torres, A., Guarda, A., Moraga, N., \& Romero, J. (2011). Experimental and theoretical study of LDPE versus different concentrations of Irganox 1076 and different thickness. Food Research International, 44(2), 566-574.

Giordano, F., Novak, C., \& Moyano, J. R. (2001). Thermal analysis of cyclodextrins and their inclusion compounds. Thermochimica Acta, 380(2), 123-151.

Greiner, A., \& Wendorff, J. H. (2007). Electrospinning: a fascinating method for the preparation of ultrathin fibers. Angewandte Chemie International Edition, 46(30), 5670-5703.

Grimme, S. (2006). Semiempirical GGA-type density functional constructed with a long-range dispersion correction. Journal of Computational Chemistry, 27(15), 1787-1799.

Hohenberg, P., \& Walter, K. (1964). Inhomogeneous electron gas. Physical Review, 136(3B), B864.

Kayaci, F., \& Uyar, T. (2012). Encapsulation of vanillin/cyclodextrin inclusion complex in electrospun polyvinyl alcohol (PVA) nanowebs: prolonged shelf-life and high temperature stability of vanillin. Food Chemistry, 133(3), 641-649.

Kfoury, M., Landy, D., Ruellan, S., Auezova, L., Greige-Gerges, H., \& Fourmentin, S. (2016). Determination of formation constants and structural characterization of cyclodextrin inclusion complexes with two phenolic isomers: carvacrol and thymol. Beilstein Journal of Organic Chemistry, 12(1), 29-42.

Kohn, W., \& Sham, L. J. (1965). Self-consistent equations including exchange and correlation effects. Physical Review, 140(4A), A1133.

Kresse, G., \& Furthmuller, J. (1996a). Efficient iterative schemes for ab initio totalenergy calculations using a plane-wave basis set. Physical Review B, 54(16), 11169.

Kresse, G., \& Furthmuller, J. (1996b). Efficiency of ab-initio total energy calculations for metals and semiconductors using a plane-wave basis set. Computational Materials Science, 6(1), 15-50.

Li, Z., Wang, M., Wang, F., Gu, Z., Du, G., Wu, J., \& Chen, J. (2007). Г-Cyclodextrin: a review on enzymatic production and applications. Applied Microbiology and Biotechnology, 77(2), 245.

López-de-Dicastillo, C., Jordá, M., Catalá, R., Gavara, R., \& Hernández-Munoz, P. (2011). Development of active polyvinyl alcohol/ $\beta$-cyclodextrin composites to scavenge undesirable food components. Journal of Agricultural and Food Chemistry, 59(20), 11026-11033.

Madigan, M. T., Clark, D. P., Stahl, D., \& Martinko, J. M. (2010). Brock biology of microorganisms (13th ed.). Benjamin Cummings.

Mallardo, S., De Vito, V., Malinconico, M., Volpe, M. G., Santagata, G., \& Di Lorenzo, M. L. (2016). Poly(butylene succinate)-based composites containing
$\beta$-cyclodextrin/D-limonene inclusion complex. European Polymer Journal, 79, 82-96.

Mathew, K., Sundararaman, R., Letchworth-Weaver, K., Arias, T. A., \& Hennig, R. G. (2014). Implicit solvation model for density-functional study of nanocrystal surfaces and reaction pathways. The Journal of Chemical Physics, 140(8), 084106.

Mourtzinos, I., Kalogeropoulos, N., Papadakis, S. E., Konstantinou, K., \& Karathanos, V. T. (2008). Encapsulation of nutraceutical monoterpenes in $\beta$-cyclodextrin and modified starch. Journal of Food Science, 73(1), S89-S94.

Mulinacci, N., Melani, F., Vincieri, F. F., Mazzi, G., \& Romani, A. (1996). 1H-NMR NOE and molecular modelling to characterize thymol and carvacrol $\beta$-cyclodextrin complexes. International Journal of Pharmaceutics, 128(1-2), 81-88.

Perdew, J. P., Chevary, J. A., Vosko, S. H., Jackson, K. A., Pederson, M. R., Singh, D. J., \& Fiolhais, C. (1992). Atoms, molecules, solids, and surfaces: Applications of the generalized gradient approximation for exchange and correlation. Physical Review B, 46(11), 6671.

Petrosyan, S. A., Rigos, A. A., \& Arias, T. A. (2005). Joint density-functional theory: ab initio study of $\mathrm{Cr} 2 \mathrm{O} 3$ surface chemistry in solution. The Journal of Physical Chemistry B, 109(32), 15436-15444.

Ramakrishna, S., Fujihara, K., Teo, W. E., Lim, T. C., \& Ma, Z. (2005). An introduction to electrospinning and nanofibers (Vol. 90) Singapore: World Scientific.

Samperio, C., Boyer, R., Eigel, W. N., III, Holland, K. W., McKinney, J. S., O’Keefe, S. F., ... Marcy, J. E. (2010). Enhancement of plant essential oils' aqueous solubility and stability using alpha and beta cyclodextrin. Journal of Agricultural and Food Chemistry, 58(24), 12950-12956.

Szejtli, J. (1998). Introduction and general overview of cyclodextrin chemistry. Chemical Reviews, 98(5), 1743-1754.

Tao, F., Hill, L. E., Peng, Y., \& Gomes, C. L. (2014). Synthesis and characterization of $\beta$ cyclodextrin inclusion complexes of thymol and thyme oil for antimicrobial delivery applications. LWT-Food Science and Technology, 59(1), 247-255.

Uyar, T., Hacaloglu, J., \& Besenbacher, F. (2009). Electrospun polystyrene fibers containing high temperature stable volatile fragrance/flavor facilitated by cyclodextrin inclusion complexes. Reactive and Functional Polymers, 69(3), $145-150$.

Uyar, T., Hacaloglu, J., \& Besenbacher, F. (2011). Electrospun polyethylene oxide (PEO) nanofibers containing cyclodextrin inclusion complex. Journal of Nanoscience and Nanotechnology, 11(5), 3949-3958.

Uyar, T., Nur, Y., Hacaloglu, J., \& Besenbacher, F. (2009). Electrospinning of functional poly (methyl methacrylate) nanofibers containing cyclodextrinmenthol inclusion complexes. Nanotechnology, 20(12), 125703.

Vega-Lugo, A. C., \& Lim, L. T. (2009). Controlled release of allyl isothiocyanate using soy protein and poly (lactic acid) electrospun fibers. Food Research International, 42(8), 933-940.

Wen, P., Zhu, D. H., Feng, K., Liu, F. J., Lou, W. Y., Li, N., \& Wu, H. (2016). Fabrication of electrospun polylactic acid nanofilm incorporating cinnamon essential oil/ $\beta$ cyclodextrin inclusion complex for antimicrobial packaging. Food Chemistry, 196, 996-1004.

Wen, P., Zhu, D. H., Wu, H., Zong, M. H., Jing, Y. R., \& Han, S. Y. (2016). Encapsulation of cinnamon essential oil in electrospun nanofibrous film for active food packaging. Food Control, 59, 366-376. 